\title{
CARÁCTER INNOVADOR DE LOS PROYECTOS DE INVESTIGACIÓN EN ENSEÑANZA DE LA FÍSICA EN EL IPN
}

\section{INNOVATOR CHARACTER OF THE RESEARCH PROJECTS IN THE TEACHING OF PHYSICS IN THE IPN}

Mario H. Ramirez Díaz

Liliana Suárez Téllez

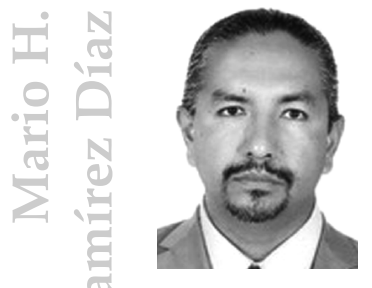

Licenciatura en Física y Matemáticas, ESFMIPN. Maestría en Ciencias con Especialidad en Física, ESFM-IPN. Doctorado en Ciencias con Especialidad en Física Educativa, CICATAIPN. Actualmente profesor titular de tiempo completo en el programa de posgrado en Física-Educativa, CICATA-IPN. Ha sido profesor del CFIE, ESCOM y ESIME Zacatenco en el IPN, UNITEC, ITESM y UNAM. Miembro del Sistema Nacional de Investigadores del CONACyT. Sus principales líneas de investigación son los estilos de aprendizaje, competencias en la enseñanza de la Física y Sociofísica. SNI. Correo electrónico: [mramirezd@ipn.mx].

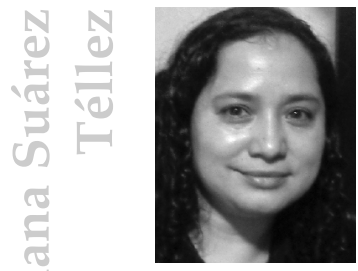

Maestría y Doctorado en Ciencias en Matemática Educativa, Centro de Investigación y de Estudios Avanzados del Instituto Politécnico Nacional (CINVESTAVIPN). Posdoctorado en la Universidad de Victoria, British Columbia, Canadá. SNI. Correo electrónico: [lsuarez@ipn.mx].

\section{RESUMEN}

El Instituto Politécnico Nacional de México (IPN), como una institución de educación técnica y tecnológica tiene, como una de sus fortalezas, la enseñanza de la ciencia y la tecnología, razón por la cual, la investigación orientada a su enseñanza es fundamental para el IPN. 
La introducción de la noción de innovación educativa en la estructura educativa del IPN ha llevado a la redefinición de muchos de sus planes y programas de estudio. En este trabajo se presenta un análisis de este carácter reformador, a partir de una serie de criterios de innovación educativa, de las propuestas de investigación en el IPN, del período 2003-2008, examinando como caso de estudio los protocolos orientados a investigar la enseñanza de la Física. Se realiza un análisis de los criterios de innovación educativa más recurrentes, proponiendo explicaciones de la razones de esta frecuencia. Finalmente, se elaboran recomendaciones a partir de los resultados analizados sobre la introducción de los criterios de innovación en los proyectos de investigación en el IPN.

Palabras clave: investigación educativa; innovación educativa; Física.

\section{ABSTRACT}

The National Polytechnic Institute, IPN as one institution of technical and technological education in Mexico has as one of his strengths science and technology teaching. As a result, the research oriented to teaching in these areas is critical to the IPN. The introduction of notion of educational innovation in the educational structure of the IPN has led to the redefinition of many of their plans and programs of study. This paper presents an analysis of innovative character, from a kit educational innovation criteria, contained in research proposals in the IPN during the period 2003-2008, taking as a case of study protocols designed to investigate the teaching of Physics. To be an analysis of the criteria for recurrent educational innovation, offering explanations of the reasons for this frequency. Finally, recommendations are made based on the results analyzed on the introduction of the criteria of innovation in research projects in the IPN.

Key words: education research; education innovation, Physics.

\section{INTRODUCCIÓN}

Desde su creación, el IPN ha otorgado una importancia fundamental a la investigación, lo cual se refleja incluso en sus documentos 
rectores (IPN, 2004). Aunque la investigación educativa en el IPN, inicia formalmente actividades en los años 70 (Lozoya, 1999), no es sino hasta el Programa Institucional de Desarrollo 1989-1994, que se señala la necesidad de fortalecer el proceso educativo; la Secretaría Académica del IPN crea, entonces, el Programa Institucional de Investigación Educativa. Este programa distingue entre investigación científica y tecnológica, e investigación educativa. Y es a partir del año 2003 que se lanzan convocatorias independientes para solicitar registro de proyectos de investigación institucionales, a través de la Coordinación General de Posgrado e Investigación, hoy Secretaría de Investigación y Posgrado (SIP) (Ramírez y Rasilla, 2006).

Por lo que se refiere al Modelo Educativo del IPN, el papel de la innovación se enfatiza en tres rubros: como un aspecto de la sociedad del conocimiento; como una de las capacidades a considerar en la formación de los estudiantes, y como una estrategia institucional para lograr sus objetivos (Ortega, et al., 2007).

Existen varios puntos de encuentro entre la investigación y la innovación, orientadas a la educación. La investigación educativa se realiza o contrata por quienes se encuentran directamente involucrados en el quehacer educativo: desde el diseño de las grandes políticas, pasando por el financiamiento de proyectos innovadores, hasta quienes desean probar innovaciones curriculares o metodológicas en el aula (Schmelkes, 2001). Existen varios tipos de investigación vinculada directamente a la innovación educativa:

a) La investigación que se realiza para diseñar innovaciones.

b) Un tipo similar de investigación directamente relacionada con la innovación educativa es vinculado a la intervención educativa directa.

c) Un tercer tipo de investigación educativa directamente ligada a la innovación es, sin duda, la evaluación.

Es importante considerar los diferentes criterios para calificar a una innovación educativa como tal. Ejemplo de lo anterior es la Guía de Evaluación de Proyectos de Innovación Pedagógica elaborada en Perú (FONDEP, 2008), que considera 17 indicadores de innovación agrupados 
en seis criterios: carácter innovador; consistencia del proyecto; metas de impacto en los aprendizajes; articulación de componentes; monitoreo y evaluación; y, finalmente, sostenibilidad.

Dentro del IPN, el Centro de Formación e Innovación Educativa (CFIE) realizó, en 2006, un instrumento para evaluar proyectos de innovación educativa derivados del observatorio politécnico de innovación educativa. Sin embargo, el IPN no considera aún alguna convocatoria oficial para realizar proyectos de innovación educativa. Como se mencionó, al momento, el IPN sólo considera convocatorias para proyectos de investigación educativa. En este trabajo se abordan precisamente estos proyectos de investigación educativa para evaluar sus criterios de innovación, tomando como caso de estudio particular los proyectos de investigación educativa orientados a la enseñanza de la Física. Se presentarán los criterios que aparecen con mayor frecuencia, explicando por qué unos son más frecuentes que otros y, finalmente, proporcionando una serie de recomendaciones sobre los proyectos de investigación.

\section{METODOLOGÍA}

\subsection{Proyectos de investigación educativa en la enseñanza de la Física}

El Consejo Mexicano de Investigación Educativa (COMIE), reúne a investigadores del país con el objetivo central de promover la investigación educativa dentro de los estándares más altos de calidad. Al elaborar el estado del conocimiento de las ciencias naturales en México, el COMIE reconoce dos grandes campos: didáctica de las ciencias y educación en ciencias, entendiendo como estado del conocimiento:

[...] el análisis sistemático y la valoración del conocimiento y de la producción generada en torno a un campo de investigación durante un período determinado (López y Mota, A., 2006).

Además, parte del supuesto de que este campo se constituye con los aportes de varias disciplinas, sin identificarse completamente con ninguna de ellas; entre estas disciplinas, incluye a la Física. 
Para realizar el estado del conocimiento de las ciencias naturales (donde se incluye la Física), el COMIE establece temáticas desprendidas de los manuales internacionales (Tobin, K., 1998): currículo, aprendizaje, teorías, historia y filosofía de la ciencia y ambientes de aprendizaje, evaluación y equidad (López y Mota, A., 2006).

Basada en estas propuestas del COMIE, desde 2003 la SIP del IPN anuncia su convocatoria para proyectos de investigación educativa ${ }^{1}$, de manera independiente y de forma paralela a la convocatoria de proyectos de investigación científica y tecnológica.

Una diferencia fundamental entre las convocatorias de investigación científica y tecnológica e investigación educativa (más allá de las evidentes) se encuentra en los recursos asignados a cada investigación. Mientras que los proyectos de investigación educativa tienen un tope de alrededor de los 1,500 USD anuales como recurso a ejercer, los proyectos de investigación científica y tecnológica pueden llegar a ejercer como máximo 5,200 USD ${ }^{2}$. Esta situación provoca que, en muchas ocasiones, los investigadores prefieran aplicar en la convocatoria de proyectos de investigación científica y tecnológica, a pesar de que sus proyectos claramente sean de investigación educativa (Ramírez y Olvera, 2010).

El presente trabajo muestra un análisis del carácter innovador de las propuestas de investigación en el IPN (período 2003-2008), a partir de una serie de criterios de innovación educativa, tomando como caso de estudio los protocolos orientados a investigar la enseñanza de la Física. Para lo anterior, lo primero fue buscar proyectos de investigación en el IPN (propuestos como de investigación educativa o de investigación científica y tecnológica) que cumplieran con estar orientados a investigar la enseñanza de la Física.

En principio, el ideal para comenzar una búsqueda del tipo de proyectos mencionados anteriormente deberían ser aquellos que han aplicado para la convocatoria de proyectos de investigación educativa de la SIP. Sin embargo, como también se mencionó, muchos proyectos

\footnotetext{
Se puede consultar la convocatoria en: [www.investigación.ipn.mx].

Al tipo de cambio de enero de 2010, en México.
} 
que bien podrían aplicar en la convocatoria de investigación educativa, prefieren ser ingresados en la convocatoria de proyectos de investigación científica y tecnológica debido a la gran diferencia en los recursos asignados en cada convocatoria. De manera que la búsqueda de proyectos no se restringió solamente a la convocatoria de investigación educativa, sino que se amplió a ambas convocatorias. Esta búsqueda se llevó a cabo en el Sistema de Administración de Programas y Proyectos de Investigación del IPN (SAPPI), y no se limitó únicamente a los proyectos «aprobados» en el período a estudiar; se analizaron todas las propuestas de proyecto de investigación o propuestas de estudios, incluyendo las rechazadas.

La búsqueda de los proyectos se basó en los protocolos ingresados por los investigadores del IPN, en el período 2003-2008, debido a que desde 2003 ambas convocatorias se consideran independientes.

Para entrar a las bases de datos del SAPPI, se solicitó permiso a la SIP y se permitió el mismo acceso que tiene un evaluador de proyecto de investigación ${ }^{3}$, es decir, acceso a los protocolos y los informes técnicos ingresados por los investigadores en el propio sistema.

Básicamente, para seleccionar los proyectos se revisaron, en los protocolos, el título, el resumen y los objetivos. Con base en la información se seleccionaron los proyectos que, a criterio de los autores de este trabajo, contaban con elementos educativos y se orientaban a la Física, sin importar si dichos proyectos solicitaron registro en la convocatoria de investigación educativa.

Dicha clasificación no deja de ser subjetiva, sobre todo por el hecho de que al no ingresar los protocolos como de investigación educativa no se asegura que el investigador en realidad quisiera proporcionarle ese enfoque, a pesar de que el resumen y los objetivos parecieran indicarlo.

Del resultado de esta búsqueda se encontraron 41 proyectos de investigación en el período 2003-2008 que se consideraron orientados al aprendizaje de la Física (Ramírez y Olvera, 2010).

3 Cabe señalar que Mario H. Ramírez y Liliana Suarez (autores de este trabajo) han sido evaluadores de proyectos de investigación educativa para la SIP, en los años 2007, 2008 y 2009. 
Como se mencionó en su convocatoria para proyectos de investigación educativa, el IPN intentó seguir las temáticas sugeridas por el COMIE. Sin embargo, en el cuerpo del protocolo no se refiere a ellas, por lo que resulta subjetivo clasificar a los proyectos de investigación en algunas de las líneas. Además, como se indicó también, no todos los proyectos estudiados en este trabajo aplicaron en la convocatoria de investigación educativa, lo cual dificultaba aún más el tratar de clasificarlos en algunas de las líneas institucionales. No obstante, y a pesar de la subjetividad implícita, a partir del análisis del título, resumen, objetivos y producto final reportados en los protocolos, se clasificaron los proyectos en alguna de las cinco áreas temáticas. Los resultados se muestran en la siguiente tabla:

\begin{tabular}{|lc|}
\hline Área temática & Número de proyectos \\
\hline Ambientes de aprendizaje & 24 \\
\hline Diseño curricular & 3 \\
\hline Administración educativa & 2 \\
\hline Formación docente & 1 \\
\hline Métodos de aprendizaje & 11 \\
\hline
\end{tabular}

Tabla 1. Número de proyectos por área temática.

El número de propuestas de investigaciones aprobadas, en comparación con las rechazadas, es muy bajo: alrededor del 30\%. Las razones de este rechazo son diversas y no son generales. Como se mencionó, la gran diferencia de recursos asignados a los proyectos de tipo educativo es un factor importante para la falta de motivación de los investigadores para incursionar en este tipo de investigación.

Se muestra que la mayoría de los proyectos del tipo educativo orientados a la Física se encuentran en el nivel bachillerato. Sin embargo, la mayoría de los profesores de este nivel en el IPN poseen una escasa formación estricta en investigación.

Además, los profesores encargados de impartir Física en el bachillerato carecen también, en muchos casos, de preparación formal para su enseñanza. 
Los dos factores anteriores — falta de preparación para realizar investigación y deficiencia en los conocimientos para enseñar Física-, impactan directamente en la realización de proyectos de investigación orientados a la enseñanza de la Física.

\subsection{Innovación educativa}

Como se mencionó en la introducción de este trabajo, para el IPN la innovación educativa ha tomado un importante rol en los últimos años. Muestra de lo anterior es la creación, en 2005, del CFIE y la elaboración del Modelo de Innovación Educativa para el IPN (CFIE, 2005). Por un lado el CFIE:

Coordinará la construcción, funcionamiento y actualización de la base de conocimientos relativos a la formación del personal y a la investigación e innovación educativa del IPN (IPN, 2009).

Específicamente en el área de Innovación Educativa el CFIE:

Es el encargado de formular y desarrollar objetivos, políticas, procedimientos y estrategias para la integración de los programas de fomento a la Investigación e Innovación Educativas, en el marco de los Modelos Educativo y de Integración Social.

Se encarga de supervisar el desarrollo de materiales educativos con tecnología que promuevan la innovación en las acciones formativas del Centro, de formular estrategias de difusión y promoción de los programas de fomento a la investigación e innovación educativas, así como darles seguimiento y promover el establecimiento de alianzas de comunidades de conocimiento y redes de colaboración internas y externas, para operar los programas de Fomento a la Investigación e Innovación Educativas a nivel nacional e internacional ${ }^{4}$.

Además, el Modelo de Innovación Educativa proporciona un marco para realizar auténticas innovaciones y para la formación y el desarrollo de una cultura de la innovación. Precisa las condiciones necesarias para que las innovaciones puedan llegar hasta la institucionalización e interiorización (Ortega, et al., 2007).

4 Para mayores detalles, consultar: [www.cfie.ipn.mx]. 
En el Modelo de Innovación Educativa del IPN se formulan doce criterios para caracterizar una innovación educativa. Estos criterios se basan en los criterios de la Red Innovemos de la UNES$\mathrm{CO}$ y en los que proponen Blanco y Messina (2000) en su Estado del arte en América Latina, publicado por la UNESCO y el Convenio Andrés Bello.

\begin{tabular}{|l|l|}
\hline CRITERIO & \multicolumn{1}{c|}{ DESCRIPCIÓN } \\
\hline Novedad: & $\begin{array}{l}\text { Una innovación introduce algo nuevo en una situación determi- } \\
\text { nada, algo nuevo que propicia una mejora con respecto a una } \\
\text { situación bien definida. No es necesario que sea una invención, } \\
\text { pero conlleva una manera diferente de configurar y vincular los } \\
\text { elementos que son objeto de la innovación. }\end{array}$ \\
\hline Intencionalidad: & $\begin{array}{l}\text { La innovación posee un carácter intencional; es un cambio que } \\
\text { deliberadamente propone lograr una mejora, una intención } \\
\text { congruente con el marco institucional. }\end{array}$ \\
\hline Interiorización: & $\begin{array}{l}\text { La innovación implica una aceptación y apropiación del cambio } \\
\text { por parte de quienes han de llevarlo a cabo. Como punto de par- } \\
\text { tida, es necesario un acuerdo entre los responsables de la } \\
\text { innovación en cuanto a los objetivos que persiguen. } \\
\text { En términos individuales, los cambios corresponden a un desa- } \\
\text { rrollo de la personalidad, en el ser, el saber y el hacer, en un pro- } \\
\text { ceso de interiorización de la innovación. Para la institución, se } \\
\text { traduce en cambios en la cultura organizacional. }\end{array}$ \\
\hline Creatividad: & $\begin{array}{l}\text { Considera explícitamente el surgimiento de iniciativas y la dispo- } \\
\text { sición para identificary yefinir problemas resolubles en todo pro- } \\
\text { ceso. La creatividad se refleja en la capacidad para identificar } \\
\text { mejoras, fijar metas y diseñar estrategias que aprovechen los } \\
\text { recursos disponibles para lograrlas. }\end{array}$ \\
\hline
\end{tabular}




\begin{tabular}{|c|c|}
\hline CRITERIO & DESCRIPCIÓN \\
\hline Sistematización: & $\begin{array}{l}\text { La innovación es una acción planeada y sistemática que involucra } \\
\text { procesos de evaluación y reflexión crítica acerca de la práctica y } \\
\text { la innovación misma. La sistematización del proceso de innova- } \\
\text { ción permite generar información contextualizada que sirve de } \\
\text { base para lo toma de decisiones fundamentales. }\end{array}$ \\
\hline Profundidad: & $\begin{array}{l}\text { La innovación genera cambios en las concepciones, actitudes y } \\
\text { prácticas educativas. Implica una auténtica transformación, una rup- } \\
\text { tura de los equilibrios aceptados en las estructuras que constituyen } \\
\text { el funcionamiento rutinario, la situación que se desea cambiar. } \\
\text { La innovación no sólo produce cambios profundos en las instala- } \\
\text { ciones, sus procedimientos y materiales, también transforma a los } \\
\text { actores educativos, sean éstos personas o sujetos colectivos. }\end{array}$ \\
\hline Pertinencia: & $\begin{array}{l}\text { La innovación es pertinente al contexto socioeducativo. El contexto } \\
\text { se reconoce como un factor esencial en el éxito de las innovaciones, } \\
\text { por lo que deben tomarse en cuenta sus características, haciendo de } \\
\text { la innovación una solución a una problemática bien definida. }\end{array}$ \\
\hline $\begin{array}{l}\text { Orientada a } \\
\text { los resultados: }\end{array}$ & $\begin{array}{l}\text { La innovación no es un fin en sí misma, sino un medio para lograr } \\
\text { mejor los fines de la educación. Los resultados de un sistema edu- } \\
\text { cativo son diversos, pero pueden destacarse la formación, la } \\
\text { generación de conocimiento y la vinculación con la sociedad. Las } \\
\text { innovaciones, entonces, producirán una mejora en los aprendiza- } \\
\text { jes de los alumnos, en la gestión del conocimiento, en los proyec- } \\
\text { tos conjuntos y en los convenios concretos establecidos entre } \\
\text { escuelas e industrias, gobierno y organizaciones sociales. }\end{array}$ \\
\hline Permanencia: & $\begin{array}{l}\text { Se ha mantenido durante el tiempo necesario para convertirse en } \\
\text { la nueva normalidad. La complejidad de la innovación implica cam- } \\
\text { bios en distintos niveles que requieren de tiempos diversos para } \\
\text { que ocurran y se consoliden. Para que estos cambios se constitu- } \\
\text { yan en la nueva normalidad, interiorizándose en las personas e ins- } \\
\text { titucionalizándose en el sistema, existe una relación no lineal entre } \\
\text { la consolidación de los cambios y el tiempo transcurrido. Sin embar- } \\
\text { go, la institucionalización de una innovación no constituye una } \\
\text { garantía de permanencia porque los procesos no son reversibles. }\end{array}$ \\
\hline
\end{tabular}




\begin{tabular}{|c|c|}
\hline CRITERIO & DESCRIPCIÓN \\
\hline Anticipación: & $\begin{array}{l}\text { En una innovación educativa se vislumbra claramente cuáles son } \\
\text { las características de la situación a lograr. Sin embargo, debe con- } \\
\text { siderarse que las innovaciones educativas - como resultado de } \\
\text { un proceso interactivo en el que pueden surgir imprevistos- sue- } \\
\text { len seguir caminos diferentes en la práctica. Cuando los objetivos } \\
\text { son claros, pueden definirse problemas nuevos en el trayecto y } \\
\text { resolverlos para seguir, de manera flexible, tratando de lograr el } \\
\text { cambio deseado pero con la disposición de modificar los términos } \\
\text { de la innovación cuando los cambios en el contexto así lo exigen. }\end{array}$ \\
\hline Cultura: & $\begin{array}{l}\text { La innovación produce cambios tanto en las concepciones como } \\
\text { en la práctica. Estos cambios contribuyen a formar una actitud } \\
\text { abierta a nuevos cambios, una actitud que no permita que la misma } \\
\text { innovación se vuelva rígida. } \\
\text { Otras características importantes de esta cultura son la colabora- } \\
\text { ción -como individuos y como parte de distintas redes-y la } \\
\text { capacidad de administrar los conflictos que, inevitablemente, sur- } \\
\text { gen en los procesos de innovación. Así mismo, la capacidad para } \\
\text { diagnosticar su situación, diseñar y poner en movimiento planes de } \\
\text { acción, y evaluar tanto su instrumentación como sus resultados. }\end{array}$ \\
\hline $\begin{array}{l}\text { Diversidad } \\
\text { de agentes: }\end{array}$ & $\begin{array}{l}\text { La diversidad de los agentes que participan en la red responsable } \\
\text { de la innovación, articula los esfuerzos en las diversas dimensio- } \\
\text { nes que atañen a la innovación. La colaboración de agentes } \\
\text { diversos en la red responsable es compleja y debe resolver los } \\
\text { conflictos surgidos por el contraste de perspectivas distintas, } \\
\text { pero también potencia la capacidad de la red para enfrentar } \\
\text { problemas multidimensionales que no pueden reducirse a las } \\
\text { perspectivas particulares de los individuos. }\end{array}$ \\
\hline
\end{tabular}

Los doce criterios para caracterizar una innovación educativa del Modelo de Innovación Educativa ${ }^{5}$.

5 Una explicación más detallada de estos criterios se encuentra en el Modelo de Innovación Educativa para el IPN (CFIE, 2005). 
Con estos antecedentes, el CFIE elaboró un instrumento para la evaluación de proyectos de innovación educativa. Es importante mencionar que, a la fecha, no existe ninguna convocatoria formal para la elaboración de proyectos de innovación educativa en el IPN, no obstante se han realizado esfuerzos encaminados a apoyar este tipo de proyectos por diferentes grupos dentro del mismo instituto. El instrumento diseñado tuvo como base los doce criterios descritos anteriormente y que son base del Modelo de Innovación Educativa del IPN. Este instrumento ha sido utilizado por el CFIE en diversas actividades académicas y de investigación.

Existen ejemplos de convocatorias para realizar proyectos de innovación educativa que consideran otro tipo de criterios, tales como los dirigidos a nivel secundaria por el gobierno de Perú (DINESST, 2005), en donde se evaluaron los proyectos por medio de preguntas abiertas sobre las características del proyecto y no basándose en una serie de criterios. También en el Perú se realizó la evaluación de proyectos de innovación pedagógica (FONDEP, 2008), la cual considera 17 indicadores agrupados en seis criterios: carácter innovador; consistencia del proyecto; metas de impacto en el aprendizaje; articulación de componentes; monitoreo y evaluación, y sostenibilidad.

En este trabajo se retomó el instrumento diseñado por CFIE, el cual se complementó agregando las características de los proyectos de investigación del IPN (anexo 1). Es importante recordar que los proyectos estudiados en esta investigación son proyectos de investigación (educativos o científico tecnológicos, como se señaló en la sección anterior), por lo tanto no están dirigidos expresamente a la innovación educativa.

El instrumento se aplicó directamente a los 38 proyectos de investigación orientados a la enseñanza de la Física; los encargados de aplicar el instrumento fueron los autores de este trabajo quienes han sido evaluadores de los proyectos de investigación en el IPN, además de participar en diversas comisiones evaluadoras de innovación e investigación educativas ${ }^{6}$. No obstante, no debe perderse de vista que toda evaluación es subjetiva. En la siguiente sección se presentan los resultados de la aplicación del instrumento.

6 Seminario Permanente de Innovación Educativa (IPN), Observatorio Mexicano de Innovación (ANUIES), Congreso Internacional de Innovación Educativa, entre otras. 


\section{RESULTADOS}

Como se mencionó en la sección anterior, se evaluaron 41 proyectos de investigación orientados a la enseñanza de la Física en el período 2003-2008. En el instrumento se podía asignar, a cada criterio de innovación, los valores de «nada», «poco», «suficiente» o «demasiado», según el criterio del evaluador. En caso de que un proyecto tuviera una mayoría de criterios evaluados, en alguno de los valores antes mencionados, se calificaba como Proyecto de Innovación Educativa en ese nivel. Los resultados de la evaluación del carácter innovador de los proyectos —empleando los doce criterios- se presentan en la tabla 2.

\begin{tabular}{|l|l|l|l|l|}
\hline & Nada & Poco & Suficiente & Demasiado \\
\hline Novedad & $12(32 \%)$ & $17(45 \%)$ & $8(21 \%)$ & $1(3 \%)$ \\
\hline Intencionalidad & $11(29 \%)$ & $18(47 \%)$ & $9(24 \%)$ & \\
\hline Interiorización & $21(55 \%)$ & $13(34 \%)$ & $4(11 \%)$ & $1(3 \%)$ \\
\hline Creatividad & $12(32 \%)$ & $15(39 \%)$ & $10(26 \%)$ & $1 \%$ \\
\hline Sistematización & $23(61 \%)$ & $9(24 \%)$ & $6(16 \%)$ & \\
\hline Profundidad & $23(61 \%)$ & $8(21 \%)$ & $7(18 \%)$ & $1(3 \%)$ \\
\hline Pertinencia & $12(32 \%)$ & $18(47 \%)$ & $8(21 \%)$ & $1(3 \%)$ \\
\hline $\begin{array}{l}\text { Orientado a } \\
\text { Resultados }\end{array}$ & $11(29 \%)$ & $16(42 \%)$ & $10(26 \%)$ & $1(3 \%)$ \\
\hline Permanencia & $26(68 \%)$ & $7(18 \%)$ & $5(13 \%)$ & \\
\hline Anticipación & $23(61 \%)$ & $8(21 \%)$ & $6(16 \%)$ & \\
\hline Cultura & $25(66 \%)$ & $8(21 \%)$ & $4(11 \%)$ & \\
\hline $\begin{array}{l}\text { Diversidad de } \\
\text { Agentes }\end{array}$ & $30(79 \%)$ & $6(16 \%)$ & $2(5 \%)$ & \\
\hline $\begin{array}{l}\text { Proyecto de } \\
\text { Investigación- } \\
\text { Innovación }\end{array}$ & $21(55 \%)$ & $10(26 \%)$ & $7(18 \%)$ & \\
\hline
\end{tabular}

Tabla 2. Evaluación del carácter innovador de los proyectos de investigación con orientación en Física del IPN en el período 2003-2008.

La primera hipótesis de este trabajo era que, dado que los proyectos no eran explícitamente de innovación, la mayoría de los criterios serían «nada» o «poco». Sin embargo, a pesar de que se comprueba la hipótesis, un porcentaje importante de proyectos (18\%) tuvo un nivel suficiente de innovación. Resulta interesante el alto nivel que tuvieron algunos criterios: «novedad», «intencionalidad», «creatividad» y «orientado a resultados», con porcentajes mayores al 20\%. Por otro lado, los criterios con porcentaje mayor en «nada innovadores» están: permanencia, cultura y diversidad de agentes, con porcentajes arriba del $65 \%$. Finalmente, un solo proyecto puede considerarse que tenía 
criterios «demasiado innovadores», aunque finalmente se consideró «suficientemente innovador».

Es notorio que la gran mayoría de los proyectos (30) no manifiestan tener diversidad de agentes; los proyectos evaluados reportan únicamente profesores de su propia área en el mejor de los casos, llegando -en muchas ocasiones - a ser el director del proyecto el único participante. Por otro lado, la gran mayoría de los proyectos no mostró poder ser permanente (26); esto puede explicarse debido al hecho de que el IPN considera, dentro de los proyectos, dos tipos: a largo plazo - tres años- y a corto plazo - un año-. En el caso de los proyectos estudiados, todos manifestaron ser de corto plazo, lo cual es un factor importante para no considerarlos permanentes. Un factor que refuerza lo anterior es que, al mismo tiempo, la mayoría de los proyectos se pueden considerar orientados a resultados (26, entre suficientes y poco), esto al ser de corto plazo y estar obligados a presentar resultados en un tiempo breve.

Los proyectos presentan, en un alto porcentaje, algún grado de creatividad (26), situación que no esperábamos en un inicio.

De los resultados anteriores se infiere que, a pesar de no estar dirigidos directamente a la innovación educativa, los proyectos de investigación en el IPN (en particular los orientados a la enseñanza de la física) pueden aumentar su nivel de innovación si se generan estímulos y condiciones para ello. Uno de ellos - y que de hecho el IPN ha tratado de fomentar- es aumentar la diversidad de agentes que participan en los proyectos de investigación. Esto se logra haciendo proyectos en los que se tengan investigadores de más de una unidad y que sean interdisciplinarios. Por otro lado, el fomentar que los investigadores propongan proyectos a largo plazo permitirá desarrollar una cultura de la innovación y los productos de investigación serán mayores y más trascendentes.

El conocer estos criterios permitiría que los investigadores los incorporaran como parte de sus protocolos de investigación. El desconocimiento de criterios como: interiorización, intencionalidad o pertinencia, provoca que a pesar de que pueden inferirse en 
los protocolos no se profundice en ellos, y aunque la Convocatoria de Proyectos de Investigación Educativa no esté diseñada para proyectos innovadores educativos, la aplicación de los criterios pueda enriquecer los protocolos.

\section{ANÁLISIS}

De los resultados mostrados en la tabla 2 se pueden profundizar, en cada criterio de innovación, aspectos particulares derivados del nivel obtenido (nada; poco; suficiente; demasiado). A continuación se muestra el análisis de cada criterio en función de los resultados obtenidos de la aplicación del instrumento.

\subsection{Novedad}

La tabla 3, muestra los resultados para el criterio «novedad»:

\begin{tabular}{|lcccc|}
\hline & Nada & Un poco & Suficiente & Demasiado \\
\hline Novedad & $12(32 \%)$ & $17(45 \%)$ & $8(21 \%)$ & $1(3 \%)$ \\
\hline
\end{tabular}

Tabla 3. Porcentajes para el criterio «novedad».

Al inicio de esta investigación se asumía la hipótesis de que los proyectos de investigación orientados a la enseñanza de la Física no presentarían altos índices de novedad; sin embargo, es notorio que alrededor del $67 \%$ de los proyectos presentan algún grado de novedad. Los proyectos en general muestran novedad al incorporar TIC a la enseñanza de la Física, en particular cursos en línea y creación de materiales para laboratorio. Por otro lado, los proyectos que no presentan novedad son aquellos que proponen, por lo general, la elaboración de notas o prácticas de laboratorio que se incorporan a algún plan de estudios que se esté actualizando.

\subsection{Intencionalidad}

La tabla 4 muestra los resultados para el criterio «intencionalidad»: 


\begin{tabular}{|lcccc|}
\hline & Nada & Un poco & Suficiente & Demasiado \\
\hline Intencionalidad & $11(29 \%)$ & $18(47 \%)$ & $9(24 \%)$ & \\
\hline
\end{tabular}

Tabla 4. Porcentajes para el criterio «intencionalidad».

Este criterio evalúa un cambio deliberado para lograr una mejora. En el caso de los proyectos estudiados, la mayoría no buscaban explícitamente dicho cambio; un $76 \%$ de los proyectos muestran poco o nada de intencionalidad. Lo anterior puede deberse a dos razones fundamentales. En primer lugar, los proyectos estudiados son de corto plazo (un año), lo cual impide proponer investigaciones que tengan por objeto una mejora inmediata en la enseñanza de la Física. En segundo término los proyectos, en su mayoría, se inscribieron como una investigación en las líneas de Ambientes de Aprendizaje o Métodos de Aprendizaje, por lo que buscan mejoras en estas áreas; sin embargo, estas investigaciones por lo general se quedan a nivel de experiencia didáctica y no llegan a un cambio a nivel institucional.

\subsection{Interiorización}

La tabla 5 muestra los resultados para el criterio «interiorización»:

\begin{tabular}{|lcccc|}
\hline & Nada & Un poco & Suficiente & Demasiado \\
\hline Interiorización & $21(55 \%)$ & $13(34 \%)$ & $4(11 \%)$ & \\
\hline
\end{tabular}

Tabla 5. Porcentajes para el criterio «interiorización».

Este criterio evalúa aceptación y apropiación del cambio. Es claro que un $89 \%$ de los proyectos presentan «nada» o «un poco» de interiorización. Una explicación posible a este alto porcentaje se debe a que los investigadores no consideran necesario que sus proyectos generen algún cambio sustancial en las prácticas para la enseñanza de la Física (lo anterior está ligado al criterio de intencionalidad). Por parte de los investigadores, el desconocimiento acerca de la importancia de este criterio para la cultura institucional, revela la necesidad 
de dar a conocer la relevancia que posee para la institución (en este caso para el IPN) el que los profesores, investigadores y estudiantes se apropien de los cambios que ellos mismos propongan y generen en su actividad académica. Sin importar que los proyectos estudiados no sean de innovación educativa, la interiorización de los mismos es esencial para la relevancia en la institución de los proyectos, con miras a los cambios que beneficien a la comunidad de la entidad educativa.

\subsection{Creatividad}

La tabla 6 muestra los resultados para el criterio «creatividad»:

\begin{tabular}{|lcccc|}
\hline & Nada & Un poco & Suficiente & Demasiado \\
\hline Creatividad & $12(32 \%)$ & $15(39 \%)$ & $10(26 \%)$ & $1(3 \%)$ \\
\hline
\end{tabular}

Tabla 6. Porcentajes para el criterio «creatividad».

$\mathrm{Al}$ empezar la investigación, se consideró que este criterio sería bajo; sin embargo, el 65\% de los proyectos se evaluó como «un poco» o «suficientemente» creativo. La mayoría de los proyectos mostró claridad al identificar y definir problemas específicos a resolver, además de proponer estrategias para la mejora de la enseñanza de la Física. No obstante, no debe perderse de vista que la mayoría de los proyectos propone soluciones a problemas muy locales - a nivel de experiencias docentes, en casi todos los casos-, sin buscar impactar institucionalmente. La creatividad se acentuó al considerar el aprovechar los recursos disponibles en la unidad de origen de los proyectos.

\subsection{Sistematización}

La tabla 7 muestra los resultados para el criterio «sistematización»:

\begin{tabular}{|lllll|}
\hline & Nada & Un poco & Suficiente & Demasiado \\
\hline Sistematización & $23(61 \%)$ & $9(24 \%)$ & $6(16 \%)$ & \\
\hline
\end{tabular}

Tabla 7. Porcentajes para el criterio «sistematización». 
Este criterio implica procesos de evaluación y reflexión crítica. Es claro que al obtener $85 \%$ de proyectos con «nada» o «un poco» de sistematización, en muchos casos, éstos no consideran procesos de evaluación o reflexión. La mayoría de los proyectos se orientan a resultados de corto plazo o a la obtención de productos de investigación (como se examinará más adelante), pero no consideran la evaluación de los resultados conseguidos ni la reflexión sobre el impacto de los productos obtenidos en el transcurso de la investigación. Un aspecto más a considerar para la falta de sistematización es el que la totalidad de los proyectos son de corto plazo (un año); por ello, los calendarios no consideran los espacios para la evaluación y la reflexión crítica.

\subsection{Profundidad}

La tabla 8 muestra los resultados para el criterio «profundidad»:

\begin{tabular}{|lcccc|}
\hline & Nada & Un poco & Suficiente & Demasiado \\
\hline Profundidad & $23(61 \%)$ & $8(21 \%)$ & $7(18 \%)$ & \\
\hline
\end{tabular}

Tabla 8. Porcentajes para el criterio «profundidad».

Este criterio evalúa los cambios en las concepciones, actitudes y prácticas educativas, así como la transformación de los actores educativos. En el caso de los proyectos estudiados, se encuentra que $82 \%$ presentan «nada» $\mathrm{o}$ «un poco» de profundidad. El principal motivo para considerar a los proyectos en estos índices es el que, al ser ellos de corto plazo, no consideran en sus protocolos cambios sustanciales en las concepciones, actitudes y prácticas que lleven a la transformación de los actores educativos; en muchos casos se limitan a intentar cambios menores en la propia práctica, sin buscar impactar a nivel institucional. En ese sentido, esta falta de profundidad está estrechamente ligada a la falta de sistematización. 


\subsection{Pertinencia}

La tabla 9 muestra los resultados para el criterio «pertinencia»:

\begin{tabular}{|lcccc|}
\hline & Nada & Un poco & Suficiente & Demasiado \\
\hline Pertinencia & $12(32 \%)$ & $18(47 \%)$ & $8(21 \%)$ & \\
\hline
\end{tabular}

Tabla 9. Porcentaje para el criterio «pertinencia».

Este criterio evalúa la solución de una problemática bien definida en un contexto socioeducativo. En el caso de los proyectos estudiados, la totalidad definía un problema a resolver; sin embargo no todos ubicaban el problema dentro de un contexto que implicara un impacto en la institución que sirviera para el mejoramiento en la enseñanza de la Física. Al aplicar el instrumento de evaluación, resultó muy difícil realizar una clara distinción de este criterio (sobre todo en la claridad de los proyectos en su inserción dentro del contexto socioeducativo en el IPN). De ahí que no exista un nivel claramente preponderante para este criterio de innovación.

\subsection{Orientado a resultados}

La tabla 10 muestra los resultados para el criterio «orientado a resultados»:

\begin{tabular}{|lcccc|}
\hline & Nada & Un poco & Suficiente & Demasiado \\
\hline $\begin{array}{l}\text { Orientado } \\
\text { a resultados }\end{array}$ & $11(29 \%)$ & $16(42 \%)$ & $10(26 \%)$ & $1(3 \%)$ \\
\hline
\end{tabular}

Tabla 10. Porcentaje para el criterio «orientado a resultados».

Como su nombre lo indica, este criterio evalúa los resultados obtenidos por un proyecto para la mejora del aprendizaje de los estudiantes. Este criterio muestra que el $81 \%$ de los proyectos presenta algún grado de «orientación a resultados». Esta situación era de esperarse dado que el protocolo de proyectos de investigación de la SIP exige 
que obtengan productos de investigación por parte de los proyectos. Dentro de los productos que reportan los proyectos destacan básicamente la generación de conocimiento (con la creación y modernización de cursos vía las TIC) y la formación de recursos humanos para la investigación (vía los becarios y las tesis producto de la investigación). Sin embargo, resultados que vinculen a la sociedad con los proyectos o la gestión de conocimiento son prácticamente nulos en los proyectos estudiados; algo que era de esperarse con los bajos índices de pertinencia, sistematización y profundidad de los proyectos.

\subsection{Permanencia}

La tabla 11 muestra los resultados para el criterio "permanencia»:

\begin{tabular}{|lcccc|}
\hline & Nada & Un poco & Suficiente & Demasiado \\
\hline Permanencia & $26(68 \%)$ & $7(18 \%)$ & $5(13 \%)$ & \\
\hline
\end{tabular}

Tabla 11. Porcentaje para el criterio «permanencia».

Este criterio evalúa si la innovación se ha mantenido durante el tiempo suficiente para convertirse en la nueva normalidad. Es claro que el $86 \%$ de los proyectos mostraron «nada» o «un poco» de permanencia. Este bajo porcentaje abarca dos explicaciones: en primer término, los proyectos son de corto plazo y no tienen una continuidad (por lo que no se comprometen a dar seguimiento a los resultados obtenidos por sus investigaciones). En segundo lugar, la mayoría de los proyectos definen problemas a resolver muy localizados, no buscan cambiar la realidad actual de la enseñanza de la Física a nivel institucional, por lo que no modifican la normalidad institucional.

\subsection{Anticipación}

La tabla 12 muestra los resultados para el criterio «anticipación»:

\begin{tabular}{|ccccc|}
\hline & Nada & Un poco & Suficiente & Demasiado \\
\hline Anticipación & $23(60 \%)$ & $8(21 \%)$ & $6(16 \%)$ & $1(3 \%)$ \\
\hline
\end{tabular}

Tabla 12. Porcentaje para el criterio «anticipación». 
En los proyectos de todo tipo (incluyendo los de innovación) deben vislumbrarse las características de la situación que se requiere modificar o lograr. En los proyectos estudiados se encontró que el $81 \%$ de ellos tienen «nada» o «poco» de anticipación; se llegó a esta evaluación dado que la mayoría de los proyectos no son claros en sus objetivos (situación curiosa si se considera que un alto porcentaje de los proyectos definen el problema y plantean los productos y resultados a obtener). Al no considerar el contexto socioeducativo en el que pretenden desarrollar su investigación, los proyectos no anticipan imprevistos o dificultades que pudieran surgir en la práctica.

\subsection{Cultura}

La tabla 13 muestra los resultados para el criterio «cultura»:

\begin{tabular}{|lcccc|}
\hline & Nada & Un poco & Suficiente & Demasiado \\
\hline Cultura & $25(65 \%)$ & $8(21 \%)$ & $4(11 \%)$ & $1(3 \%)$ \\
\hline
\end{tabular}

Tabla 13. Porcentajes para el criterio «cultura».

Este criterio evalúa los cambios propuestos que contribuyan a formar una actitud abierta de forma que la innovación no se vuelva rígida. En el caso de los proyectos estudiados, el $86 \%$ mostró tener un nivel de «nada» o «un poco» de cultura. Era de esperarse dados los bajos resultados obtenidos en otros criterios que implican necesariamente una cultura de innovación, tales como la colaboración entre individuos (criterio de «diversidad de agentes», que se analizará más adelante), administrar los conflictos que surgen en los procesos de innovación («anticipación») o evaluar resultados («sistematización»). No obstante algunos proyectos sí mostraban que eran candidatos a crear una cultura de la innovación dentro de su ámbito de investigación para la enseñanza de la Física, considerando los aspectos mencionados anteriormente. 


\subsection{Diversidad de agentes}

La tabla 14 muestra los resultados para el criterio «diversidad de agentes»:

\begin{tabular}{|lccll|}
\hline & Nada & Un poco & Suficiente & Demasiado \\
\hline $\begin{array}{l}\text { Diversidad } \\
\text { de agentes }\end{array}$ & $30(79 \%)$ & $6(16 \%)$ & $2(5 \%)$ & \\
\hline
\end{tabular}

Tabla 14. Porcentajes para el criterio «diversidad de agentes».

Este criterio evalúa la diversidad de agentes que permiten la articulación de esfuerzos en las diversas dimensiones de la innovación. Es de destacar que este criterio tuvo una menor calificación con un 95\% de «nada»o «un poco». La razón principal para este bajo índice se debió a que la gran mayoría de los proyectos, al considerar en su protocolo a los integrantes, reportó en muchas ocasiones sólo al director del proyecto (79\%); en otros casos consideró a profesores participantes de la misma unidad académica, más precisamente de la misma área o academia (en este caso de Física); algunos proyectos consideraron estudiantes becarios, todos del mismo plantel donde se desarrolló el proyecto, y únicamente en dos proyectos se reportó la participación de profesores y estudiantes externos a la unidad donde se realizó el proyecto. A excepción de uno, todos los proyectos incluyeron únicamente profesores y en menor medida estudiantes; un proyecto incluyó a un directivo y personal de apoyo a la educación, de ahí la necesidad de ampliar el panorama de agentes en los proyectos, no sólo para la innovación, sino para la investigación en general.

Después del análisis anterior se encontró que 21 proyectos presentaron más de 6 criterios en nivel de «nada innovadores», 10 en nivel de «poco innovadores», 6 en nivel de «suficiente» y uno tuvo 6 criterios en «suficiente» y 6 en «demasiado». En un principio se pensó que la mayoría de los proyectos de investigación estarían en un nivel de «nada innovadores», sin embargo, un $45 \%$ de los proyectos tenían al menos 6 criterios con algún grado de innovación. No debe perderse de vista que estos proyectos no se dirigían directamente a la innovación; sin embargo también debe tenerse en cuenta que la investigación, por 
sí misma, debe ser una actividad innovadora y generar algunos de los criterios de innovación estudiados en este trabajo. De hecho, la gran mayoría de los proyectos estudiados mostraron que, con las adecuaciones pertinentes, podrían incorporar los criterios de innovación, situación que implicaría una mejora en la investigación en sí misma.

\section{CONCLUSIONES}

De los resultados conseguidos y analizados en este trabajo se obtiene, a manera de conclusiones, una serie de reflexiones sobre los proyectos de investigación en el IPN y de recomendaciones para proponer proyectos de innovación educativa en el mismo instituto.

En función de lo obtenido en la sección anterior pueden señalarse las siguientes reflexiones para los proyectos de investigación educativa estudiados en este trabajo:

- Para introducir y desarrollar de mejor manera criterios de innovación en los proyectos de investigación es necesario proponer proyectos de mediano o largo plazo (al menos tres años) de manera que se pueda tener sistematización, profundidad y cultura como resultado de la investigación. Estos criterios no sólo generarán condiciones idóneas para la innovación, sino que además mejorarán el trabajo de investigación en sí mismo.

- Los proyectos deben buscar impactar más a nivel institucional que a nivel local, definiendo la resolución de problemas que sean generales a todo el IPN, encontrando con esto una mayor intencionalidad e interiorización de los proyectos.

- Se requiere mayor precisión en los objetivos y productos de investigación de los proyectos, esto permitirá una mayor «anticipación» frente a los problemas que se encuentran en el desarrollo de la investigación, así como dirigir de manera más precisa los «resultados» esperados de la investigación.

- Debe buscarse mayor interdisciplinariedad incorporando profesores de diferentes áreas y líneas de conocimiento, así como diversificar 
a los agentes que colaboren con las investigaciones (directivos, estudiantes y personal de apoyo a la investigación). Asimismo, tener contactos con agentes externos al propio instituto con la finalidad de crear redes que permitan realizar un trabajo de investigación más integral y general.

Las reflexiones anteriores se refieren a los proyectos de investigación tal y como se tienen a la fecha; no hay que olvidar que, actualmente, el IPN no tiene una convocatoria explícita para proyectos de innovación educativa, no obstante se han hecho esfuerzos para concretar una convocatoria para este tipo de proyectos a nivel institucional. En ese sentido y en función de los resultados mostrados en este trabajo, se pueden formular las siguientes recomendaciones para una eventual convocatoria de proyectos de innovación educativa:

- Los proyectos deben tener un mínimo de 3 años (mediano plazo) de duración, de manera que puedan ser «sistemáticos», "pertinentes», «permanentes» y que creen «cultura de innovación» en la institución.

- Deben proponer objetivos claros y resultados medibles que impacten en la enseñanza en el instituto, buscando la resolución de problemas específicos y bien definidos en el IPN («anticipación», «intencionalidad», «interiorización», «orientado a resultados»).

- Deben priorizarse proyectos que consideren participantes de diferentes unidades académicas, entre profesores, estudiantes (becarios), directivos y personal de apoyo a la educación («diversidad de agentes»).

- Deben impactar en la cultura institucional proponiendo mejoras sustanciales en metas específicas («creatividad», «novedad»).

Con estas recomendaciones se cubren prácticamente los doce criterios de innovación estudiados en este trabajo. Por otro lado, una convocatoria de proyectos de innovación educativa que incorpore estas recomendaciones tendría grandes posibilidades de generar proyectos que impactaran directamente en la cultura institucional de innovación y, de mantenerse y apoyarse el tiempo necesario, podrían transformar la enseñanza del instituto. 
El carácter innovador de los proyectos de investigación permitiría (incluyendo las recomendaciones previas) una mayor participación entre estudiantes, profesores y cuerpo directivo, no sólo de una unidad académica sino institucionalmente y de forma ideal con otras instituciones nacionales y extranjeras. Esto sólo será posible en caso de que se cree una cultura de la innovación ligada con la pertinencia, situación considerada en el IPN por medio de sus documentos rectores (como el Modelo de Innovación Educativa). La socialización de resultados de investigación permitirá la creación de cuadros especializados en el área de la investigación educativa, pobre aún en el IPN; muestra de ello es que de 2003 a la realización de este estudio se encontraron sólo 38 proyectos considerados, en alguna medida, con contenidos de innovación educativa, muestra que para un estudio en general es escasa sobre todo tomando en cuenta el tamaño del IPN. Sin embargo, es importante notar esta escasez como un área de oportunidad de crecimiento en dicha rama de la investigación. En caso de no poder contar con una convocatoria para proyectos de innovación educativa podrían considerarse, para la evaluación de los proyectos de investigación (educativa o científica y tecnológica), algunos de los criterios aquí mostrados de manera que dichos proyectos se enriquecerían, toda vez que muchos de estos criterios están considerados de manera no formal por los investigadores y, al tomar conciencia de su existencia e importancia, profundizarían en el desarrollo de los mismos.

\section{Anexo 1 \\ FICHA DE EVALUACIÓN DE CRITERIOS DE INNOVACIÓN DE PROYECTOS DE INVESTIGACIÓN EN EL IPN}

Título del proyecto:

Descripción:

\section{Objetivo:}


Producto final:

Agentes:

Área temática:

Criterios de innovación educativa

\begin{tabular}{l|l|l|l|l}
\hline & Nada & Un poco & Suficiente & Demasiado \\
\hline Novedad & & & & \\
\hline Intencionalidad & & & & \\
\hline Interiorización & & & & \\
\hline Creatividad & & & & \\
\hline Sistematización & & & & \\
\hline Profundidad & & & & \\
\hline Pertinencia & & & & \\
\hline $\begin{array}{l}\text { Orientado } \\
\text { a resultados }\end{array}$ & & & & \\
\hline Permanencia & & & & \\
\hline Anticipación & & & & \\
\hline Cultura & & & & \\
\hline $\begin{array}{l}\text { Diversidad } \\
\text { de agentes }\end{array}$ & & & & \\
\hline $\begin{array}{l}\text { Proyecto de } \\
\text { Investigación- }\end{array}$ & & & & \\
\hline
\end{tabular}




\section{REFERENCIAS BIBLIOGRÁFICAS}

BLANCO, R. y MESSINA, G., Estado del arte sobre las innovaciones educativas en América Latina, Convenio Andrés Bello, Bogotá, 2000.

CFIE., Modelo de Innovación Educativa para el IPN. Estrategias y acciones para generar la Innovación Educativa, IPN, México, 2005, en: [www.eventos.cfie.ipn.mx/content/innovacion/.../modelo.pdf].

DINESST., Bases del concurso interno de proyectos de innovación educativa. Asesoría y fortalecimiento de instituciones educativas emblemáticas como líderes de la reforma de la educación secundaria, Dirección Nacional de Educación Secundaria y Superior Tecnológica, Gobierno del Perú, 2005.

FONDEP., Guía para la evaluación de proyectos de innovación educativa, Fondo Nacional de Desarrollo de la Educación Peruana, Lima, 2008.

IPN., Materiales para la reforma, programa estratégico de investigación y posgrado, IPN, México, 2004.

«Reglamento orgánico del Instituto Politécnico Nacional», Gaceta Politécnica, número extraordinario 747 bis, México, 2009.

LÓPEZ y MOTA, Saberes científicos, humanísticos y tecnológicos Tomo I: Procesos de enseñanza y aprendizaje, COMIE, México, 2006.

LOZOYA, E., La investigación educativa en el Instituto Politécnico Nacional frente al siglo XXI, IPN, México, 1999.

ORTEGA, P.; RAMÍREZ, M.; TORRES, J.; LÓPEZ, A.; SERVÍN, C.; SUÁREZ, L. y RUIZ, B., Modelo de innovación educativa. Un marco para la formación y el desarrollo de una cultura de la innovación, RIED, vol. 10: 1, 2007, p. 145-173. 
RAMÍREZ, M. y RASILLA, M., Investigación educativa en el IPN del siglo XXI, n. 6, Innovación Educativa, 2006, p. 62-67.

RAMÍREZ, M. y OLVERA, M., La investigación educativa en Física en el Instituto Politécnico Nacional de México, Lat. Am. J. Phys. Educ., 2010 (por publicar).

SCHMELKES, S., La investigación en la innovación educativa, Conferencia Magistral, Congreso Latinoamericano sobre Innovaciones Educativas, Cholula, Puebla, 2001.

TOBIN, K., «Issues and Trends in the Teaching of Science», en B. Fraser y K. Tobin (eds.), International Handbook of Science Education, vols. 1 y 2, Kluwer Academic Publishers, Dordrecht, 1998, p. 129- 151. 